\title{
Hemangioma cutáneo gigante en extremidad inferior fetal con diagnóstico ecográfico prenatal: reporte de un caso
}

\author{
Ángela Franco Sansaloni ${ }^{1}$, Carmina Bovea Beltrán ${ }^{1}$, Roser Batalla Gurrea ${ }^{1}$, Sebastián \\ Ortolá Chumillo ${ }^{1}$, Elía Villar Graullera ${ }^{1}$, César Romeo Cabrera León. ${ }^{1}$ \\ ${ }^{1}$ Adjuntos del Servicio de Obstetricia y Ginecología, Unidad de Medicina Materno-Fetal, Hospital General y Universitario \\ de Castellón. España.
}

Primer y segundo autor han colaborado de forma equitativa en la elaboración de este artículo.

\section{RESUMEN}

Antecedentes: Los hemangiomas cutáneos son unas de las anomalías congénitas más frecuentes en el recién nacido. La búsqueda bibliográfica revela que la información respecto a su diagnóstico prenatal y actitud terapéutica es muy dispersa. Caso clínico: Presentamos el diagnóstico prenatal de un hemangioma fetal de grandes dimensiones, de $63 \times 48 \mathrm{~mm}$, localizado en cara anterior del muslo fetal izquierdo que es detectado inicialmente por ecografía en la semana 28 y confirmado por resonancia nuclear magnética. Conclusiones: Planteamos las alternativas diagnósticas que surgen ante este hallazgo, así como su pronóstico y manejo.

\section{PALABRAS CLAVE: Hemangioma cutáneo gigante, diagnóstico prenatal, ecografía}

\section{SUMMARY}

Background: Cutaneous hemangiomas are a really common congenital malformation in neonates. A literature search has revealed a sparsity of information about prenatal diagnosis and management. Case report: We present the prenatal diagnosis of a giant-size cutaneous hemangioma $(63 \times 48 \mathrm{~mm})$, located in the left thigh by ultrasonography in the 28th week of pregnancy and confirmed by magnetic resonance imaging. Conclusion: We propose diagnostic possible alternatives, prognosis and management.

\section{KEY WORDS: Cutaneous hemangioma, prenatal diagnosis, ultrasonography}

\section{INTRODUCCIÓN}

Los hemangiomas son tumores vasculares benignos muy comunes dentro de las anomalías congénitas (1). La mayoría de ellos aparecen como pequeñas lesiones fundamentalmente localizadas en cabeza y cuello que pasan inadvertidas durante las exploraciones ecográficas intraútero por su insignificante tamaño, en estos casos no se acompañan de transcendencia clínica y se resuelven espontáneamente durante la infancia (2). Sin embargo, los hemangiomas gigantes sí se han visto asociados a complicaciones graves que pueden desencadenarse tanto en el periodo fetal como neonatal, tales 
como la insuficiencia cardíaca congestiva, anemia, trombocitopenia incluso el síndrome de KasabachMerrit (3), por lo que su diagnóstico prenatal comporta implicaciones a tener en cuenta para el futuro bienestar del binomio materno-fetal.

El objetivo de esta presentación es presentar el diagnóstico prenatal de un hemangioma fetal gigante en extremidad fetal izquierda realizado por ecografía, así como el manejo posterior en un hospital terciario.

\section{Caso clínico}

Primigesta de 29 años, de origen español, sin antecedentes médicos ni quirúrgicos de interés a excepción de hipotiroidismo tratado con levotiroxina 137 mg/día. Gestación actual espontánea, bien controlada de 28 semanas, con tamizaje combinado de primer trimestre informado de bajo riesgo para cromosomopatías y estudio morfológico ecográfico de segundo trimestre sin hallazgos de importancia. En la semana 28, acude para exploración ecográfica de rutina, la biometría fetal es acorde a la edad gestacional, líquido amniótico normal y placenta de inserción posterior grado 0 . Durante la evaluación fetal se visualiza una gran tumoración de consistencia sólida, homogénea de $63 \mathrm{x}$ $48 \mathrm{~mm}$ de contorno irregular en la cara anterior del muslo izquierdo, que se extiende a lo largo de toda su longitud, las estructuras óseas subyacentes no impresionan desplazadas y sus medidas son correspondientes a edad gestacional (Figura 1). En el estudio Doppler de la masa se detecta una importante vascularización, con vasos de baja resistencia con presencia de conexiones arteriovenosas. El resto de la anatomía fetal resulta compatible con la normalidad, no se identifican otras anomalías asociadas ni otras imágenes susceptibles de alteración morfológica. Nuestra primera opción como diagnóstico de sospecha de esta tumoración de tejido blando es hemangioma cutáneo gigante sin poder descartar de forma tajante otro tipo de formaciones como sarcoma o miofibromatosis congénita, por lo que se solicita resonancia nuclear magnética (RMN), sin contraste, que define la masa de $65 \mathrm{x}$ $48 \mathrm{~mm}$, de aspecto exofítico a nivel de tejido celular subcutáneo de muslo fetal izquierdo sin signos de afectación ósea ni muscular presentando características compatibles con el diagnóstico de hemangioma cutáneo fetal gigante (Figura 2).

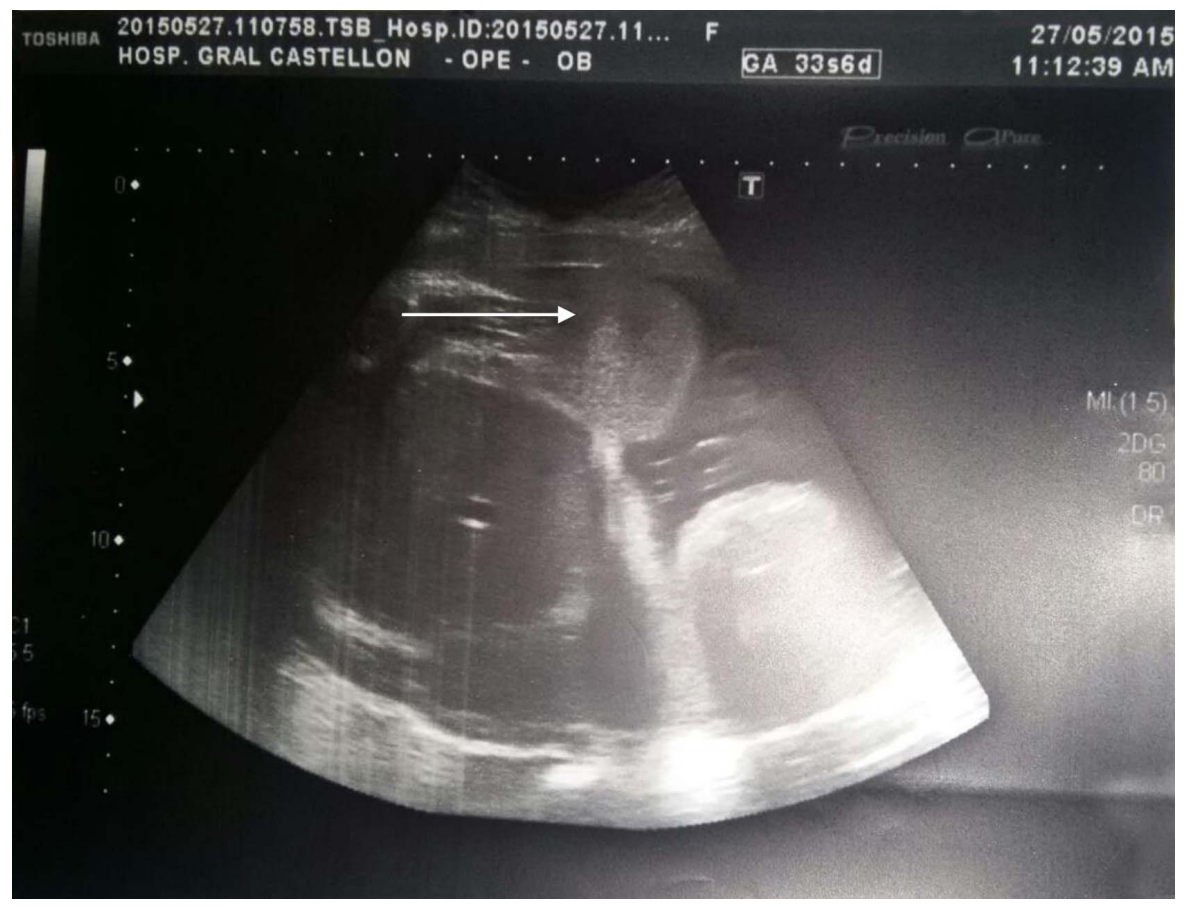

Figura 1. Corte transverso a nivel de abdomen fetal en ecografia transabdominal de seguimiento en semana 33+6. La flecha señala la porción proximal del fémur, apreciando la presencia de una masa homogenea, hipoecogénica, de aspecto sólido. 


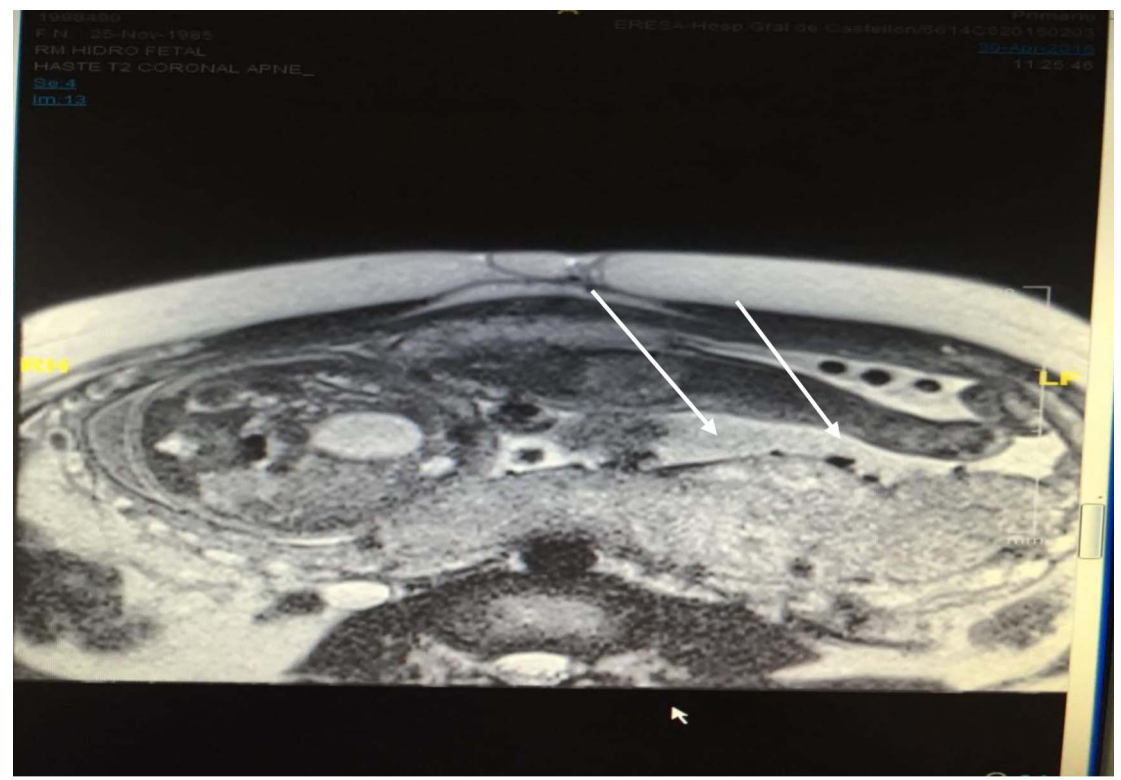

Figura 2. Resonancia magnética nuclear (RMN), sin administración de contraste, T2, obtenida en la semana 29, donde se observa una tumoración de tejido blando de 63 x $48 \mathrm{~mm}$, que se extiende sobre la total longitud del fémur.

El caso es asumido por el comité de perinatología de nuestro centro, constituido por un equipo multidisciplinar formado por 3 obstetras, 3 pediatras, 2 cirujanos pediátricos, 2 matronas; dado las posibles complicaciones asociadas a la presencia y tamaño de la masa se decide actitud expectante con controles semanales ecográficos y de bienestar fetal y finalización de la gestación por cesárea electiva en semana 37, descartando parto vía vaginal evitando con ello el riesgo de distocia, traumatismo y sangrado que éste pueda desencadenar, facilitando la presencia de cirugía pediátrica en caso de resultar necesaria.

La pareja es informada en todo momento tanto de los hallazgos como del diferente rango de gravedad que puede alcanzar el desenlace, aceptando el manejo prenatal ofrecido. El embarazo evoluciona sin eventos remarcables, durante el seguimiento no se aprecia aumento ecográfico significativo en las dimensiones de la masa ni en su vascularización, en ninguna exploración existe evidencia de ascitis ni otros signos de insuficiencia cardíaca congestiva, con crecimiento fetal acorde a la edad gestacional y ambas extremidades inferiores con movimientos espontáneos dentro de la normalidad. A las 36 semanas +5 , la gestante acude de urgencias por rotura prematura de membranas, se practica cesárea programada a las 36 semanas +6 , obteniendo un recién nacido femenino, de 2780 gramos, Apgar 9/10 sin requerir maniobras de reanimación. En la valoración neonatal se confirma la existencia de una masa de coloración violácea, rojo-vinosa, no pulsátil, de márgenes bien definidos, aspecto angiomatoso, sin ulceración, que se extiende por todo el segmento anterolateral del muslo izquierdo, sin afectar la flexo-extensión de la extremidad, de 70 x $40 \mathrm{~mm}$ de diámetro (Figura 3).

El recién nacido es trasladado a la unidad de neonatología donde se efectúa observación estrecha durante las primeras 24 horas, presentando en todo momento parámetros de estabilidad, recibiendo alta hospitalaria a las 72 horas. Se realiza tanto control ecográfico como nueva RMN en el periodo postnatal sin cambios sustanciales respecto a los detectados en las exploraciones realizadas intraútero y se decide por parte de pediatría biopsiar la lesión obteniendo confirmación anatomopatológica para hemangioma. A los 3 meses del nacimiento se evidencia cierto grado de regresión por lo que se postpone inicialmente tratamiento médico o quirúrgico, optando por actitud expectante y seguimiento trimestal por dermatología, pediatría y cirugía pediátrica. 


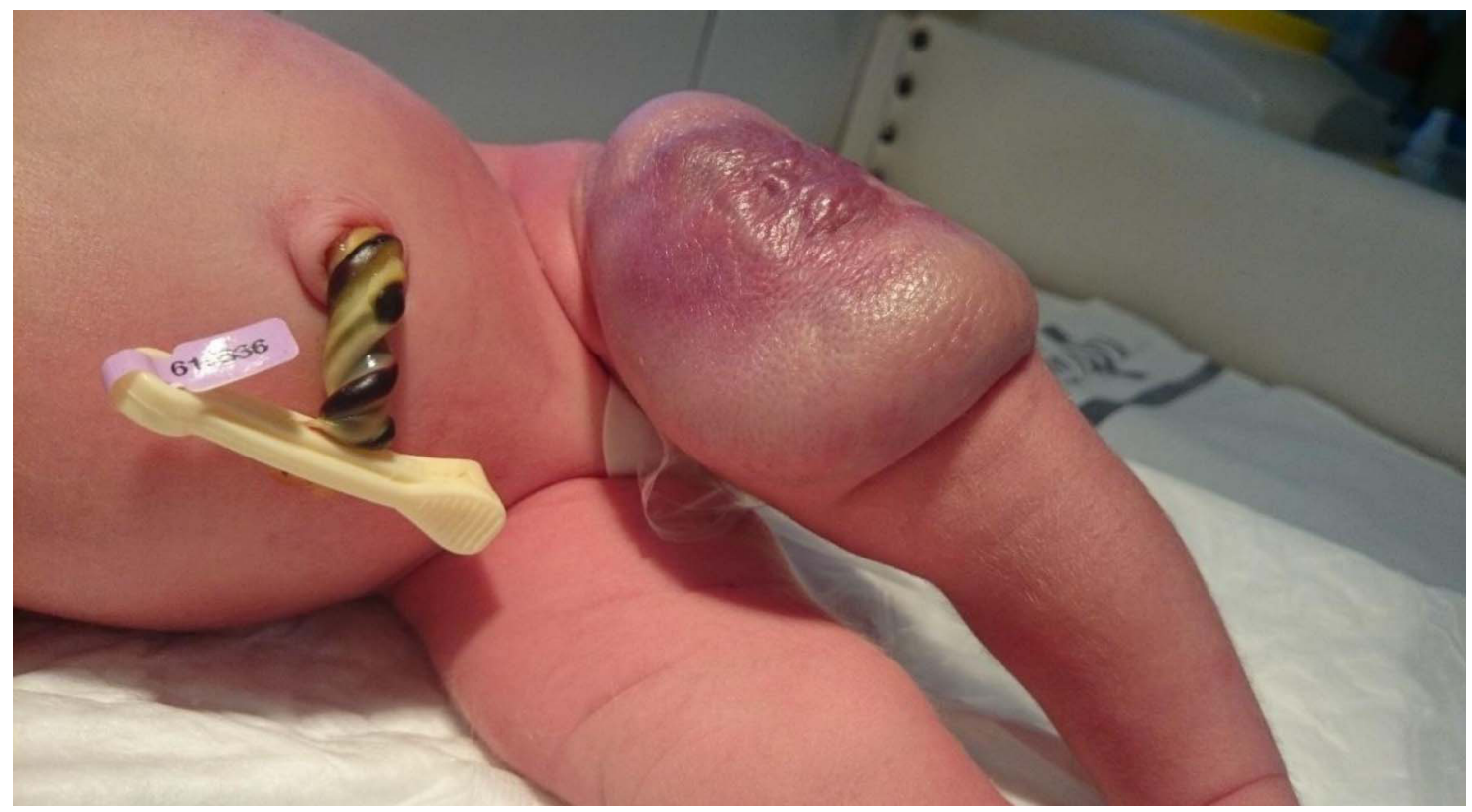

Figura 3. Fotografía postnatal, mostrando al neonato con la masa sobre la cara anterior del muslo izquierdo, compatible con hemangioma cutáneo.

\section{DISCUSIÓN}

El hemangioma es el tumor de partes blandas más común en la infancia, su frecuencia se estima entre un 5-10\% de la población menor a un año de vida, afectando mayoritariamente al sexo femenino en una proporción $4: 1$, a la raza blanca y fetos prematuros (4).

A pesar de su elevada frecuencia y a que hasta el $50 \%$ de estas lesiones se encuentran presentes en el momento del nacimiento, los casos descritos en la literatura son más bien escasos, de hecho en las últimas 2 décadas sólo encontramos en la búsqueda medline 3 casos clínicos relacionados con diagnóstico prenatal de hemangiomas gigantes en extremidad fetal $(5,6,7)$; si bien es cierto que el $80 \%$ de los hemangiomas son solitarios, de pequeño tamaño y presentan una involución espontanea, existe un $20 \%$ de hemangiomas complicados, con posibilidad de provocar graves complicaciones que comprometan la vida del recién nacido o alterar el funcionamiento permanentemente de algún órgano (8).

Los hemangiomas gigantes, como el descrito en nuestro caso, pueden ocasionar fallo cardíaco congestivo intraútero debido a una circulación hi- perdinámica compensatoria secundaria a un gasto cardíaco aumentado y pueden desencadenar la aparición de trombocitopenia, anemia hemolítica y coagulopatía de consumo (síndrome de KasabachMerrit). El sangrado, ulceración y posterior sobreinfección de la masa pueden surgir como complicaciones intra y postoperatorias, existiendo casos descritos de hemorragia severa durante la primera semana de vida $(9,10)$. Por todo ello, el diagnóstico prenatal de un hemangioma gigante es de vital importancia para efectuar un manejo adecuado del mismo, implicando no sólo al equipo obstétrico sino llevar a cabo una evaluación por un equipo multidisciplinar, con el objetivo de facilitar una monitorización fetal estrecha durante el embarazo que incluya tanto control ecográfico de la tumoración como de los parámetros de circulación fetal, selección de la mejor vía para conseguir un parto atraumático y evitar problemas derivados de la distocia por el efecto masa, como también disponer de las medidas neonatales necesarias incluyendo cirugía pediátrica, para efectuar el mejor tratamiento en caso de las serias complicaciones que puedan presentarse en el periodo postnatal inmediato, siendo recomendable programar el nacimiento en un centro hospitalario terciario. 


\section{CONCLUSIÓN}

El diagnóstico prenatal mediante ultrasonografía permite identificar tumores de partes blandas vasculares de gran volumen y así programar la vía de parto en un centro terciario para su tratamiento y seguimiento neonatal.

\section{REFERENCIAS}

1. Escribano D, Gallego M, Galindo A, Delgado MD, de la Fuente P. Diagnóstico prenatal de hemangioma cutáneo fetal. Prog Obstet Ginecol 2002;45(12):571-4.

2. Podwell J. Update on hemangiomas and vascular malformations. Curr Opin Pediatr 1999;11:457-63.

3. Treadwell MC, Sepulveda W, LeBlanc LL, Romero R. Prenatal diagnosis of fetal cutaneous hemangioma: case report and review of the literature. J Ultrasound Med 1993;12:683-7.

4. Madrigal C, Bidonga A, Madrigal V. Hemangiomas múltiples gigantes: respuesta al tratamiento con corticoides. Rev Pediatr Aten Primaria 2010;12(47):e4753.
5. Suma V, Marini A, Gamba PG, Luzzatto C. Giant hemangioma of the thigh: prenatal sonographic diagnosis. J Clin Ultrasound 1990;18:421-4.

6. Sheiner E, Gohar J, Mazor M. Prenatal diagnosis of giant hemangioma of the thigh. Int J Gynecol Obstet 1999;67:175-6.

7. Gonçalves LF, Pereira ET, Parente LM, Vitorello DA, Barbosa UC, Saab Neto JA. Cutaneous hemangioma of the thigh: prenatal diagnosis. Ultrasound Obstet Gynecol 1997;9:128-30.

8. Hering S, Sarmiento FGR, Valle LE. Actualización en el diagnóstico y tratamiento de los hemangiomas. Rev Argent Dermatol 2006;87:54-66.

9. Shuichiro $\mathrm{U}$, Toshimichi $\mathrm{H}$, Hiroomi O, Hisayoshi K, Akio K, Keigo O, Eiichi M. Prenatally detected giant congenital hemangioma of the fetal neck. J Pediatr Surg 2013;1(3):29-31.

10. Powel J, Blouin M.M, David M, Dubois J. Bleeding in congenital hemangiomas: crusting as a clinical predictive sign and usefullness of tranexemic acid. Pediatr Dermatol 2012;29:182-5. 